\title{
WHY THE SPIN-FET DOES NOT WORK?*
}

\author{
A. Dargys \\ Semiconductor Physics Institute, A. Goštauto 11, LT-01108 Vilnius, Lithuania \\ E-mail: dargys@pfi.lt
}

Received 21 March 2007

\begin{abstract}
The spin-FET (spin field effect transistor) is the spintronics device the operation of which is based on unique properties of free electron or hole spin in semiconductors. In the spin-FET, the modulation of channel conductance is achieved by control of spin direction via the spin-orbit interaction. The present article, firstly, critically reviews the main difficulties encountered in realizing the spin-FET. Secondly, using the concept of the spin surface, general spin properties of 2D holes in $p$-type channel of the FET are considered. The complex nature of the spin surfaces of the ballistic 2D holes is demonstrated. It is shown that in optimizing the spin-FET, in addition to the dispersion law of the channel one should pay a special attention on how the spin surface transforms between spin injector and collector.
\end{abstract}

Keywords: spintronics, spin-FET, hole spin, spin-orbit interaction, quantum well

PACS: 71.70.Ej, 75.10.Dg, 85.30.Tv

\section{Introduction}

Electron is an object with the charge and spin. But so far the semiconductor electronics has completely neglected the spin. In 1990, Datta and Das proposed a spin analog of the electrooptic modulator that later was renamed spin-FET [1]. In the spin-FET the source and drain are ferromagnetic materials (Fig. 1) where free carriers have a fixed spin direction. Whereas in a standard semiconducting FET the gate voltage controls the number of charge carriers in channel and, thus, modulates the current flowing through the FET, in the spinFET the gate voltage controls the spin orientation of the electrons in the channel via spin-orbit interactions and, due to drain selectivity to spin state, modulates the current flowing through the device. The spin orientation is changed by electric field via Rashba spinorbit (SO) interaction mechanism [2,3]. Since electron transmission probability at channel-drain interface depends on relative alignment of the spin with respect to the drain magnetization direction, the total electrical current appears to be dependent on the spin state. When the spin of the electron incident on the drain is aligned with the drain magnetization, the transmission is the largest and, therefore, the current flowing through the device is maximal. However, the current stops to

\footnotetext{
* The report presented at the 37th Lithuanian National Physics Conference, 11-13 June 2007, Vilnius, Lithuania.
}

flow if the spin direction changes to opposite during electron flight in the spin-FET channel. Thus, by controlling the spin precession angle between the source and the drain electrodes one can switch on or switch off the electrical current. Since the described spin manipulation scheme does not require magnetic fields, the spin-FET can be integrated in the existing charge-based semiconductor technology without incorporation of external magnetic systems. However, the attempts to realize the spin-FET till now have been unsuccessful because of a number of obstacles, both of practical and theoretical character. Below, the problems of theoretical nature related to spin superposition states will be touched in more detail, with a special accent on the 2D hole carrier spin surfaces which represent all possible directions and magnitudes of the average spin in the spin space. The properties of spin surfaces in bulk semiconductors were considered earlier in [4-9].

\section{Salient features and requirements to spin-FET}

\subsection{Principal differences between electronic and hole spin-FETs}

The discussion below will be limited to $\mathrm{A}_{3} \mathrm{~B}_{5}$ compound semiconductors the spin properties in which are most investigated. The conduction band of these semiconductors is made up predominantly of $s$-type orbitals, 


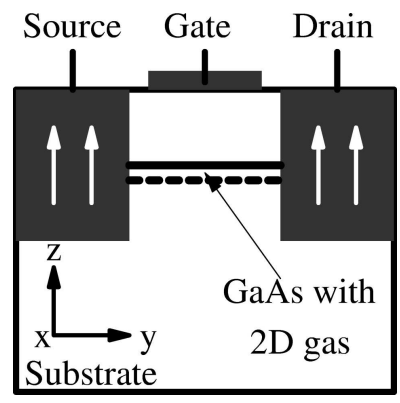

Fig. 1. Schematic drawing of the spin field effect transistor (spinFET). The quantum well (QW) containing spin-polarized 2D gas lies in the $x-y$ plane. The vertical magnetization of the ferromagnetic source and drain electrodes is shown by vertical arrows. The horizontal magnetization is possible too. The spatial polarization of the 2D electron or hole gas in the channel can be controlled by voltage applied to the gate electrode.

the SO interaction of which with the spin is indirect (via other bands) and, for this reason, is rather weak. Inclusion of the SO interaction lifts the double degeneracy of the conduction band and fixes the orientation of the quantization axis. The spin-split energy bands are characterized by opposite spin directions with respect to the quantization axis, Fig. 2. The spin-split bands are related by Kramers symmetry, $E_{\uparrow}(\mathbf{k})=E_{\downarrow}(-\mathbf{k})$, where $E_{\uparrow}(\mathbf{k})$ is the carrier energy whose wave vector is $\mathbf{k}$ and the spin is directed along the quantization axis. Normally the spin splitting energy $\Delta E=E_{\uparrow}(\mathbf{k})-E_{\downarrow}(\mathbf{k})$ is small, about (1-10) meV. If the spin splitting is due to Rashba interaction [2] (the main process used to control the spin orientation in semiconductors), the conduction band splitting depends linearly on the wave vector:

$$
\Delta E_{\mathrm{e}}=2 \alpha_{\mathrm{e}} k,
$$

where $\alpha_{\mathrm{e}}$ is the Rashba constant and $k=\sqrt{k_{x}^{2}+k_{y}^{2}}$ is the wave vector in the plane of the QW. Similar dependence was found for 2D holes that originate from the 3D light-mass valence band. The consequence of the proportionality of $\Delta E_{\mathrm{e}}$ to $k$ is that the spin precession length (see Eq. (7)) is independent of the carrier energy. Thus, all injected electrons or light-mass holes will experience the same precession angle during their ballistic flight from the source to drain electrode. However, in case of $p$-type QWs, usually, the principle energy band has a heavy-mass rather than light-mass character. It can be shown that in the latter case the dependence of the splitting energy on $k$ (at least at small $k$ values) is cubic rather than linear [10]:

$$
\Delta E_{\mathrm{HH}}=2 \alpha_{\mathrm{HH}} k^{3} .
$$

Thus, for heavy 2D holes it will be more difficult to guarantee equal precession lengths of the ensemble of the holes if the initially injected holes are spread in energy.

However, the fundamental difference between the electrons and holes comes from their spin properties. In a free space, as known, the spin surface of a nonrelativistic electron is the Bloch sphere [11]. For a free conduction band electron in $\mathrm{A}_{3} \mathrm{~B}_{5}$ compounds the spin surface is spherical too, if the conduction band is spindegenerate. Inclusion of the SO interaction has very small influence on the sphericity. Calculations show that the electron spin surface in these compounds deviates from the sphere by less than $1 \%$ [12]. This explains why the evolution of the average electron spin $\langle\mathbf{S}\rangle$ in $\mathrm{A}_{3} \mathrm{~B}_{5}$ compounds can be described by precession equation that coincides with the classical equation of the top:

$$
\frac{\mathrm{d}\langle\mathbf{S}\rangle}{\mathrm{d} t}=-\boldsymbol{\Omega} \times\langle\mathbf{S}\rangle,
$$

where $\boldsymbol{\Omega}$ is the precession vector. The module of $\Omega$ is equal to the conduction band spin splitting energy (1). Because of the simplicity of spin dynamics on the spherical surface, the bulk of papers in semiconductor spintronics are devoted to analysis of electronic spin-FETs [13]. In the 2D channels that originate from the valence band, as will be shown in the next section, the spin surfaces strongly deviate from the spherical shape. This is due to strong SO interaction in the $A_{3} B_{5}$ semiconductor valence bands, which are predominantly made up of $p$-orbitals. The same was found to be true for bulk holes in semiconductors. Examples of spin trajectories on spin surfaces of bulk semiconductors can be found in Ref. [8].

The simplicity of electron spin surfaces suggests that they could serve as a benchmark in answering the principal questions in spintronics, at least theoretically. However, the comparison of theory with experiment at present is difficult due to lack of good spin injecting contacts. At present, the experimentalists work with a circularly polarized light to generate spin-polarized electrons in semiconductors [14]. Since during excitation both the polarized electrons and holes are generated, in order to have only polarized electrons their spin lifetime should be long compared to the hole spin lifetime. In the case of $\mathrm{A}_{3} \mathrm{~B}_{5}$ semiconductors this is easy to achieve, because complex valence band structure favours short spin coherence times of free holes. On the other hand, the experimental situation with hole spin injection is more encouraging. The diluted magnetic semiconductors, for example, GaAs heavily doped with Mn impurities, have been shown to be an 


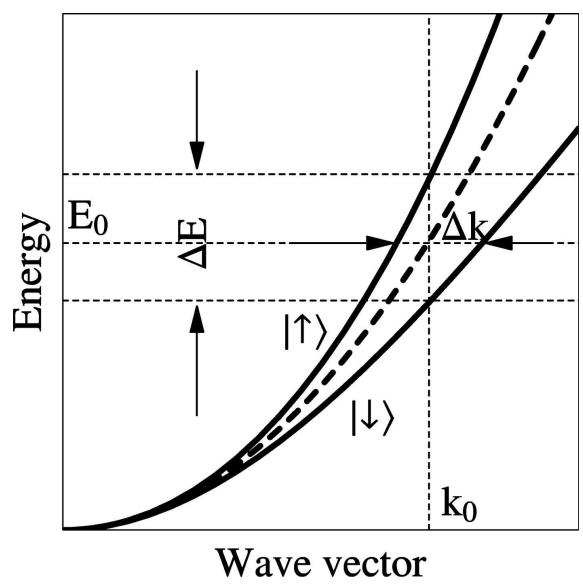

Fig. 2. The dashed line shows the dispersion of a doubly spindegenerate band. The SO interaction splits this band into "up" and "down" spin bands (solid lines) with respect to quantization axis. The bands in this figure are inverted and represent the heavy-mass hole band (cf Fig. 3). The important parameters are $\Delta E$ and $\Delta k$ which determine the magnitude of spin splittings along the energy and wave vector axes at the Fermi energy $E_{0}$. The splitting $\Delta k$ determines spatial spin precession and the pitch of a helix trajectory drawn by a tip of the spin along the channel. $\Delta k$ should be large to have nanometre or shorter channel lengths. The splitting $\Delta E$ describes the spin precession as a function of time at a fixed point in the channel. The condition $\Delta E \gg k T$ should be satisfied for a proper operation of the spin-FET.

efficient source of the spin-polarized holes [15]. However, the commercial applicability of the diluted magnetic semiconductors in microelectronic technologies, in our case in fabrication of spin source and drain electrodes, requires high Curie temperatures. The current record is $173 \mathrm{~K}$ in GaAs: Mn epilayers. As an alternative one can use transition metals. However, the injectors fabricated from these metals are less efficient and are more difficult to match with the semiconductor material technologically, although they allow to reach higher Curie temperatures.

\subsection{Spin superposition states and precession length}

For a proper operation of the spin-FET the electron should be in a superposition of up and down spin states. In the dispersion represented by two solid lines in Fig. 2 this corresponds to two points on the respective lines. The resulting spinor is

$$
\begin{aligned}
|\psi(y, t)\rangle= & \mathrm{e}^{\mathrm{i}\left(\omega_{0}+\Delta \omega / 2\right) t+\mathrm{i}\left(k_{0}+\Delta k / 2\right) y} \cos \vartheta|\uparrow\rangle \\
& +\mathrm{e}^{\mathrm{i}\left(\omega_{0}-\Delta \omega / 2\right) t+\mathrm{i}\left(k_{0}-\Delta k / 2\right) y} \mathrm{e}^{\mathrm{i} \phi} \sin \vartheta|\downarrow\rangle,
\end{aligned}
$$

where the coordinate $y$ runs along the channel, Fig. 1 . In (4) it was assumed that the splittings $\Delta k / 2$ and $\Delta \omega / 2=\Delta E / 2 \hbar$ are symmetric with respect to the degeneracy point $\left(k_{0}, \omega_{0}\right)$, where $\omega_{0}=E_{0} / \hbar$. The spinor (4) is normalized. The parameters $\vartheta$ and $\phi$ allow to vary the contribution of spin-up $|\uparrow\rangle$ and spin-down $|\downarrow\rangle$ eigenvectors in the mixed state. At $\vartheta=0$ and $\vartheta=\pi / 2$ one regains pure up and down states. As will be shown in section 3 with the help of exact quantum mechanical solution, the true quantization axis in the QW with Rashba interaction included is parallel to $x$ axis. Then, using the Pauli matrices $\boldsymbol{\sigma}=\left(\sigma_{x}, \sigma_{y}, \sigma_{z}\right)$ transformed so that the spin quantization axis lies along $x$ axis, $\boldsymbol{\Sigma}=\mathbf{U}^{\dagger} \boldsymbol{\sigma} \mathbf{U}$, where $\mathbf{U}=1 / \sqrt{2}\left(\begin{array}{cc}1 & -1 \\ 1 & 1\end{array}\right)$, and the spinor (4), one finds the following average spin:

$$
\begin{aligned}
\langle\mathbf{S}(y, t)\rangle= & \langle\psi(y, t)|\mathbf{\Sigma}| \psi(y, t)\rangle= \\
& \frac{1}{2}(\cos 2 \vartheta \\
& -\sin (\Delta \omega t+\Delta k y-\phi) \sin 2 \vartheta \\
& -\cos (\Delta \omega t+\Delta k y-\phi) \sin 2 \vartheta)
\end{aligned}
$$

When $\vartheta=\pi / 4$, it is seen that the parameter $\phi$ fixes the initial spin polarization which should be set to $\phi=\pi$ in the case of vertical polarization and to $\phi=\pi / 2$ in the case of horizontal polarization. For an arbitrary $\phi$ and $\vartheta=\pi / 4$ the spin lies in the $y-z$ plane. In addition, if one has $\Delta \omega=\Delta E / \hbar=0$ (see Fig. 2) in the superposition state, from Eq. (5) it follows that the average spin is time-independent and at the distance $y=L$ it makes up some fixed angle with the $z$ axis,

$$
\langle\mathbf{S}(L, t)\rangle=\frac{1}{2}(0, \sin \Delta k L, \cos \Delta k L) .
$$

The equation (6) describes a helix as a function of the distance $L$. The pitch of the helix (precession length) can be controlled by external electric field via $\Delta k$, i. e. via the spin splitting of the bands. The initial and final spins should be either parallel or antiparallel in the conducting and nonconducting state respectively [1]. If $\Delta \omega \neq 0$, there, in addition, will be a rotation of the spin around the quantization axis with frequency $\Delta \omega$. This is undesirable, since the direction of the spin at the drain electrode will not be fixed in space. Thus, at the finite lattice temperature $T$, the condition $\Delta \omega \gg k T$ should be satisfied in order to minimize the contribution of mixed states having different energies. At room temperature, due to small spin splitting in $\mathrm{A}_{3} \mathrm{~B}_{5}$ semiconductors $\Delta E=(1-10) \mathrm{meV}$ [16], this is difficult to satisfy.

The spin precession length is given by $L_{\mathrm{SO}}=$ $2 \pi / \Delta k$. For electrons and light-mass holes, as 
mentioned, the precession length is independent of carrier energy and is given by [1]

$$
L_{\mathrm{SO}}^{\mathrm{e}}=L_{\mathrm{SO}}^{\mathrm{LH}}=\frac{2 \pi}{\alpha_{\mathrm{e}, \mathrm{LH}}} \frac{\hbar^{2}}{2 m_{\mathrm{e}, \mathrm{LH}}},
$$

where $\alpha_{\mathrm{e}, \mathrm{LH}}$ and $m_{\mathrm{e}, \mathrm{LH}}$ are the Rashba coefficient and the effective mass for electrons or light-mass holes, respectively. For holes that originate from the heavymass band the spin splitting is given by Eq. (2) and the precession length decreases with hole energy [17],

$$
L_{\mathrm{SO}}^{\mathrm{HH}}=\frac{2 \pi}{\alpha_{\mathrm{HH}}}\left(\frac{\hbar^{2}}{2 m_{\mathrm{HH}}}\right)^{2} \frac{1}{E_{0}} .
$$

For typical values, $\alpha_{\mathrm{e}, \mathrm{LH}} \sim 0.01 \mathrm{eV} \mathrm{nm}$ and $\alpha_{\mathrm{HH}} \sim$ $0.005 \mathrm{eV} \mathrm{nm}^{3}$, the expressions (7) and (8) give $L_{\mathrm{SO}}^{\mathrm{e}} \approx$ $L_{\mathrm{SO}}^{\mathrm{LH}} \approx 240 \mathrm{~nm}$ and $L_{\mathrm{SO}}^{\mathrm{HH}} \approx 150 \mathrm{~nm}$ at Fermi energy $E_{0}=0.05 \mathrm{eV}$. The mean free paths of the ballistic carriers must be longer than the above evaluated precession lengths. This condition is the most difficult to satisfy in the case of holes, especially at high temperatures when optical phonon scattering becomes important. Experiments show that the mean free path of the injected holes may be as long as $300 \mathrm{~nm}$ at low temperatures [18]. Of course, at high temperatures the ballistic length will be smaller.

\subsection{The dephasing of spinor}

Collisions of the carrier with impurity atoms, defects, and phonons may change the direction of the ballistic spin. The coherence distance determines the minimum length of channel that allows to fulfil on and off conditions in the spin-FET. To have a maximum modulation depth of the spin-dependent current the injected charge carrier should not experience the collisions which destroy coherence of the spinor. In semiconductors, usually the spin coherence or relaxation time due to collisions with the impurities and phonons is comparable to or longer than momentum relaxation time. There is a large number of papers devoted to spin relaxation in semiconductors, thus, only the review article [13] and the paper [19] are mentioned here for reference. In general, it has been found that the electron spin dephasing time is longer than the momentum scattering time and, therefore, the dephasing is not a limiting factor. However, in the case of holes the situation is worse. Here the efficient spin relaxation is related to the strong SO interaction and the emission of optical phonons when the hole energy is high enough. For example, optical orientation experiments with undoped bulk GaAs at room temperature [20] give that due to optical phonon emission the hole spin lifetime can be as short as $0.11 \mathrm{ps}$. At low temperatures the spin lifetime, in general, is substantially longer and is not a limiting factor. However at room temperature the role of optical phonons remains important in 2D as well as 3D structures.

As mentioned above, for an efficient operation of the spin-FET the carrier must be in a superposition state with $\Delta E=0$, Fig. 2. At finite device temperature there will also be superpositions with different energies in the energy range $k T$. Such superpositions give spins precessing with different frequencies at the channeldrain interface and, as a result, the leakage current appears in the device. This reduces the contrast between on and off states. At present the condition $\Delta E \ll k T$ can be satisfied at low temperatures only since Rashba constants in different materials are too small [16].

\subsection{Differences between $1 D$ and $2 D$ spin-FETs}

In 2D FET the carrier is bounded in one direction and can move freely in two perpendicular directions. As a result there is an unavoidable angular spread of the injected carriers in the channel. In a flat electrode configuration this yields different mean free paths and spin rotation angles at the drain electrode. In 1D spinFET the confinement of a moving carrier is maximal, and this eliminates the angular spread that is always present in 2D channels. This was already noted in the original paper by Datta and Das [1]. This is important since in the off-state the 1D spin-FET will have no leakage current due to angular spread and, thus, the standby power dissipation will be avoided. Moreover, in the 1D channel there is a possibility to eliminate or to avoid major spin relaxation mechanisms. Therefore, the 1D channel is always more advantageous with respect to 2D channel [21].

\subsection{Semiconductor-ferromagnetic interface}

As shown in Refs. [22, 23], the double spin refraction appears at the interface separating the injector and 2D gas in the channel. The refraction follows from both the conservation of the electron wave vector parallel to the interface and the spin splitting of the energy bands. As shown by Pala et al. [17], the double spin refraction should be taken into account to get correct boundary condition for the total spinor at the spin injector (collector) and 2D channel interface. The double spin refraction allows one to calculate the coherence length of the spinor in absence of spin scattering events since it allows to take correctly into account the 
interference due to different injection angles and different source-drain distances. The finite spin coherence length is limiting the useful channel length because the incoherence causes the leakage current that may be unacceptably large in the off state. The calculations [17] show that due to this mechanism the coherence length in $2 \mathrm{D}$ channels is about (2-3) $L_{\mathrm{SO}}$ in the case of holes. To minimize the effect of double refraction on the leakage current it is desirable to have lateral dimensions of the channel as small as possible. In this respect, 1D channels are preferable.

Since the spin-FET is supposed to work in the ballistic regime ${ }^{1}$, the reflection of the spinor from the semiconductor-ferromagnetic interfaces also may have an effect on injection as well as on collection efficiencies in the on-state. The reflection coefficient depends on both the injector/ collector and the channel properties which include many factors, such as the double refraction and the deviation of the average spin from optimal direction given by Eq. (6). In addition, FabryPerot type resonances may develop between the injecting and collecting electrodes and this may enhance or suppress the electrical current in the FET. In the paper [25] the interplay between the Fabry-Perot resonance and Rashba-Dresselhaus spin splitting mechanism has been used to simulate possible modulation of the spin conductance when the source and drain electrodes are not magnetized at all. In the next section it will be shown that some insight into the properties of the spin-FET may be gained if one also takes into account the structure of the spin surfaces on both sides of the interfaces. The problem of the spin surface is much simpler than the full quantum mechanical problem. Since the shape of the spin surface is insensitive to $\Delta E$ and $\Delta k$, at least when conditions $\Delta E \ll E_{0}$ and $\Delta k \ll k_{0}$ are satisfied, the problem can be simplified further by neglecting the SO interaction at all and treating the degenerate bands only.

\subsection{Role of the quantization axis}

The quantization axis is undefined and has no privileged direction if the energy bands are spin degenerate. The magnetic field $\mathbf{B}$ induces spin splitting of the doubly degenerate bands and at the same time fixes the direction of the quantization axis, usually along $\mathbf{B}$ in

\footnotetext{
${ }^{1}$ There were proposals to relax strictly ballistic transport by using the interplay between Rashba and Dresselhaus SO interaction mechanisms [24]. The strength of these mechanisms can be tuned so that the spinor becomes independent of the carrier wave vector even in two dimensions. Vanishing of the wave vector nullifies the scattering matrix element if the bands are parabolic.
}

the absence of other spin-related mechanisms. In semiconductors that do not possess the inversion symmetry the quantization axis in absence of the magnetic field is fixed by SO interaction (Rashba, Dresselhaus), crystal symmetry, and to some extent by properties of the confining potential. If, in addition, the magnetic field is applied, the direction of the quantization axis will also depend on $\mathbf{B}$, although now it will not necessarily be aligned with $\mathbf{B}$. The knowledge of the direction of quantization axis and $\mathrm{k}$ allows one to do some predictions about the free charge carrier spin properties. The "up" and "down" energy bands in Figs. 2 and 3 are measured with respect to this effective quantization axis. However, to find the direction of the quantization axis one has to solve full quantum mechanical problem. In some cases the quantization axis can be guessed from heuristic considerations. Then spin properties can be predicted from simpler spin-degenerate Hamiltonians.

\subsection{In- and out-of-plane electrode magnetization}

Usually in the analysis the magnetization directions in both spin injecting and collecting electrodes are assumed to be identical, since technologically it is easier to produce identical electrodes. Two geometries are possible: when the magnetization vector lies in the plane of QW, or when the magnetization is perpendicular to QW plane as shown in Fig. 1. These magnetization geometries should be matched with the direction of the quantization axis of a ballistic carrier that propagates along the channel. The calculations show that for electrons as well as for holes the quantization axis appears perpendicular to carrier wave vector and at the same time lies in the QW plane if only the Rashba SO interaction is accounted for. If one imagines that the quantization axis and the wave vector are attached to the moving carrier, then the knowledge of $\Delta E$ and $\Delta k$ allows one to portrait the variation of the spin as a function of time and position in the channel.

\section{Spin surfaces in $p$-type $2 \mathrm{D}$ channels}

\subsection{Hamiltonian and spectrum}

The heuristic formula (5) yields spherical spin surface. The average spin length $|\langle\mathbf{S}\rangle|$ given by (5) is independent of band mixing parameters $\vartheta$ and $\phi$. In the case of the valence band a stricter treatment of the problem is required. The valence band Hamiltonian that 
includes the quadratic $H_{\mathrm{LK}}$ and linear $H_{\mathrm{SO}}$ in the wave vector terms will be considered:

$$
H_{\mathrm{v}}=H_{\mathrm{LK}}+H_{\mathrm{SO}}
$$

Four band Luttinger-Kohn (LK) Hamiltonian will be used [26],

$$
H_{\mathrm{LK}}=\left[\begin{array}{cccc}
H_{\mathrm{hh}} & b & c & 0 \\
b^{*} & H_{\mathrm{lh}} & 0 & c \\
c^{*} & 0 & H_{\mathrm{lh}} & -b \\
0 & c^{*} & -b^{*} & H_{\mathrm{hh}}
\end{array}\right],
$$

where

$$
\begin{aligned}
H_{\mathrm{hh}}= & \frac{\hbar^{2}}{2 m_{0}}\left[\left(\gamma_{1}+\gamma_{2}\right)\left(k_{x}^{2}+k_{y}^{2}\right)\right. \\
& \left.-\frac{\partial}{\partial z}\left(\gamma_{1}-2 \gamma_{2}\right) \frac{\partial}{\partial z}\right]+V(z), \\
H_{\mathrm{lh}}= & \frac{\hbar^{2}}{2 m_{0}}\left[\left(\gamma_{1}-\gamma_{2}\right)\left(k_{x}^{2}+k_{y}^{2}\right)\right. \\
& \left.-\frac{\partial}{\partial z}\left(\gamma_{1}+2 \gamma_{2}\right) \frac{\partial}{\partial z}\right]+V(z), \\
b= & -\mathrm{i} \frac{\sqrt{3} \hbar^{2}}{2 m_{0}}\left(-k_{y}-\mathrm{i} k_{x}\right)\left(\gamma_{3} \frac{\partial}{\partial z}+\frac{\partial}{\partial z} \gamma_{3}\right), \\
c= & \frac{\sqrt{3} \hbar^{2}}{2 m_{0}}\left[\gamma_{2}\left(k_{x}^{2}-k_{y}^{2}\right)-2 \mathrm{i} \gamma_{3} k_{x} k_{y}\right] .
\end{aligned}
$$

Here $m_{0}$ is the free electron mass, and $\gamma_{1}, \gamma_{2}$, and $\gamma_{3}$ are the Luttinger valence band parameters that depend on coordinate $z . V(z)$ describes the potential profile of the barriers and the quantum well: $V(z)=V_{0}$ if $z$ is in the barrier, $0 \leq z<\left(L_{\mathrm{t}}-L_{\mathrm{w}}\right) / 2$ or $\left(L_{\mathrm{t}}+L_{\mathrm{w}}\right) / 2 \leq z<L_{\mathrm{t}}$, and $V(z)=0$ if $z$ is in the well, $\left(L_{\mathrm{t}}-L_{\mathrm{w}}\right) / 2 \leq z<\left(L_{\mathrm{t}}+L_{\mathrm{w}}\right) / 2$, where $L_{\mathrm{w}}$ is the well width and $L_{\mathrm{t}}$ is the total length of the structure (channel). $V_{0}$ is the height of the barrier. To include the boundary conditions, the Hamiltonian $H_{\mathrm{LK}}$ was symmetrized with respect to coordinate $z$.

The contribution of hole states to the spin splitting due to structural inversion asymmetry was approximated by a matrix $[3,10]$

$$
H_{\mathrm{SO}}=r_{1}\left[\begin{array}{cccc}
0 & \frac{\sqrt{3}}{2} k_{-} & 0 & 0 \\
\frac{\sqrt{3}}{2} k_{+} & 0 & k_{-} & 0 \\
0 & k_{+} & 0 & \frac{\sqrt{3}}{2} k_{-} \\
0 & 0 & \frac{\sqrt{3}}{2} k_{+} & 0
\end{array}\right],
$$

where $k_{ \pm}=k_{x} \pm k_{y}$. The Hamiltonians (10) and (15) are written for the following order of the projections of total angular momentum of the hole: $m_{J}=(+3 / 2,+1 / 2,-1 / 2,-3 / 2)$. In calculations the Rashba interaction strength, which differs from the averaged one in the Eq. (8), was assumed to be $r_{1}=$ $0.005 \mathrm{eV} \mathrm{nm}$. Two depths of the well were considered, $V_{0}=0.0956 \mathrm{eV}$ and $V_{0}=0.604 \mathrm{eV}$, which correspond to $\mathrm{Ga}_{0.21} \mathrm{Al}_{0.79} \mathrm{As} / \mathrm{GaAs}$ and AlAs / GaAs wells. The valence band parameters for respective materials are: for GaAs, $\gamma_{1}=6.85, \gamma_{2}=2.1, \gamma_{3}=2.9$; for AlAs, $\gamma_{1}=3.45, \gamma_{2}=0.68, \gamma_{3}=1.29$; for $\mathrm{Ga}_{0.21} \mathrm{Al}_{0.79} \mathrm{As}$, $\gamma_{1}=6.14, \gamma_{2}=1.8, \gamma_{3}=2.56$. In all cases the QW width $L_{\mathrm{w}}=100 \AA$ and the total widths $L_{\mathrm{t}}=300 \AA$ were used.

Figure 3 shows the dispersions of the first three 2D energy bands for (a) shallow and (b) deep QWs. At $k=0$ the Hamiltonian (9) is diagonal and, as a result, the energies are doubly degenerate. At $k \neq 0$ the band splitting is induced by SO Hamiltonian (15). The magnitude of the splitting $\Delta E=E_{\uparrow}(\mathbf{k})-E_{\downarrow}(\mathbf{k})$ is shown in Fig. 4 for shallow well. For deep well the dependence is very similar. It is seen that the relations (1) and (2) are satisfied at small splitting energies only. It should be noted that in the intermediate energy range the heavy hole splitting is proportional to $k$. However, at high energies the deviation from both linear and quadratic dependences is strong.

\subsection{Spin surfaces}

The spin surfaces were determined from average spin

$$
\langle\mathbf{S}\rangle=\langle\psi|\mathbf{S}| \psi\rangle,
$$

where $\mathbf{S}=\left(S_{x}, S_{y}, S_{z}\right)$ and $S_{i}$ is one of the Cartesian $4 \times 4$ Pauli-like matrices. In the considered approximation $\mathbf{S}=\mathbf{J} / 3$, where $\mathbf{J}$ is the total angular momentum matrix with the component $J_{z}$ diagonal [4]. To draw 


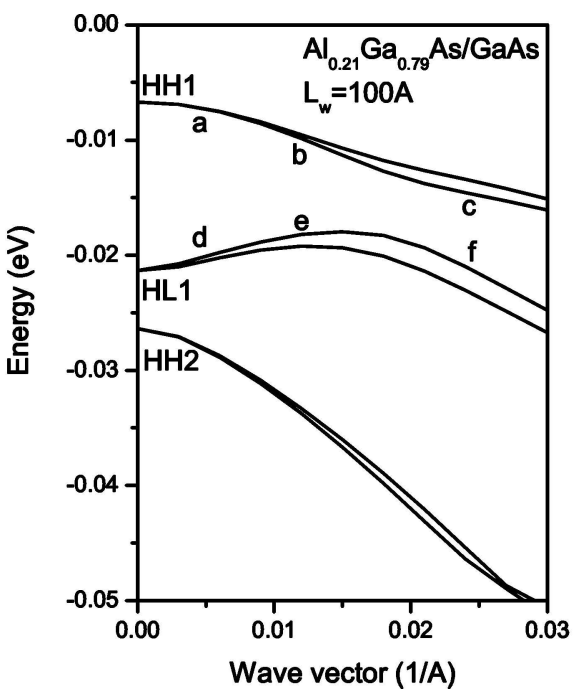

(a)

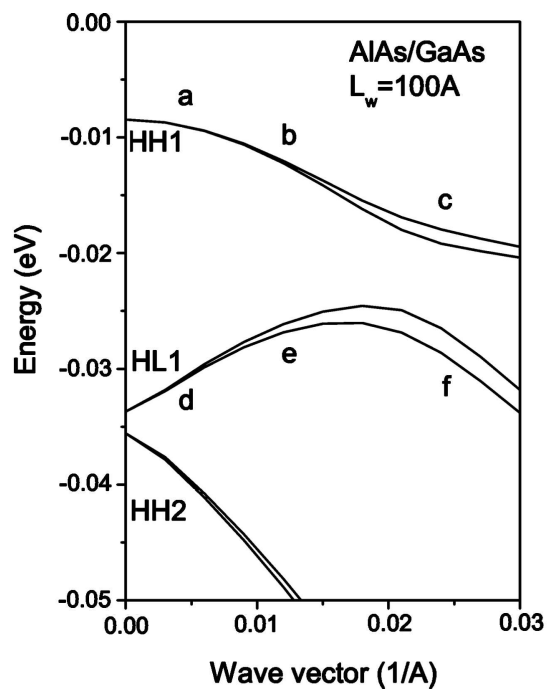

(b)

Fig. 3. Dependence of hole energy on the wave vector parallel to [01] direction. Only three main subbands degenerate at $k=0$ are shown. The well depth in (a) is $0.0956 \mathrm{eV}$ and in (b) it is $0.604 \mathrm{eV}$. The letters on curves correspond to respective panels in Figs. 5 and 6.

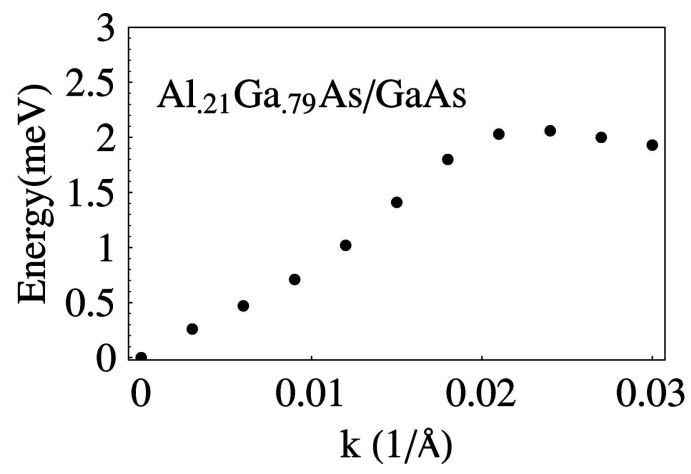

(a)

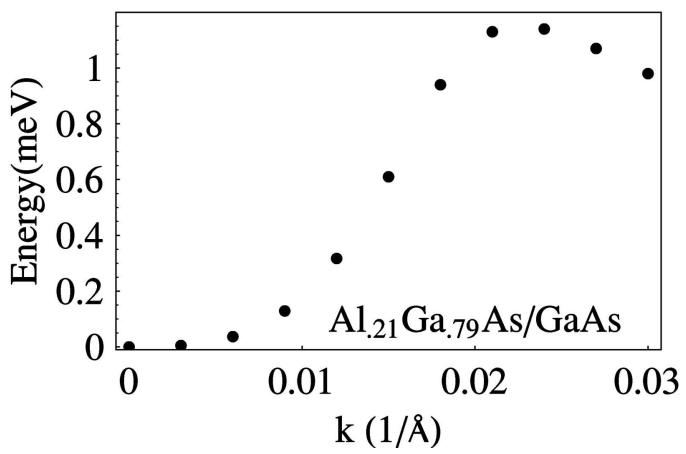

(b)

Fig. 4. Dependence of the spin splitting energy $\Delta E$ on hole wave vector for (a) HL1 and (b) HH1 bands. The well depth is $0.0956 \mathrm{eV}$.

the spin surface, the wave function $|\psi\rangle$ was parameterized:

$$
|\psi\rangle=\cos \vartheta\left|\psi_{\uparrow}\right\rangle+\sin \vartheta \mathrm{e}^{\mathrm{i} \phi}\left|\psi_{\downarrow}\right\rangle,
$$

where $\left|\psi_{\uparrow}\right\rangle$ and $\left|\psi_{\downarrow}\right\rangle$ are the eigenstates of the Hamiltonian (9) with opposite spins but with the same energy, $E\left(k_{\uparrow}\right)=E\left(k_{\downarrow}\right)$. To satisfy the latter equation, the wave vectors $k_{\uparrow}$ and $k_{\downarrow}$ must be different, Fig. 2. By varying the parameters $\vartheta$ and $\phi$ one can change the direction and magnitude of the average spin leaving the energy of the hole fixed. Alternatively, one can calculate the eigenstates at different energies, $E\left(k_{\uparrow}\right) \neq E\left(k_{\downarrow}\right)$, but at the same wave vector, $k_{\uparrow}=k_{\downarrow}$. Since spin splitting is small, the difference in spin surfaces calculated by both methods was found to be negligibly small. From the computational point of view the second method is simpler.

Figures 5 and 6 show spin surfaces of the two lowest energy subbands at three wave vectors parallel to [01] direction. The corresponding wave vectors are also indicated on the dispersion curves by letters in Fig. 3 . First of all, it should be noted that, in general, the surfaces are not spherical. In fact they are spheroids. The ratio of large and small spheroid axes is sensitive to the magnitude of the wave vector. When the wave vector is rotated in the QW plane, the spin surface synchronously rotates about $\left\langle J_{z}\right\rangle$ axis, however, its overall shape remains nearly the same. Secondly, the two poles (at $\vartheta=0$ and $\pi / 2$ ) on the spin surfaces correspond to two orthogonal eigenstates in (17) and are representatives of the spin-split energy subbands with up and down spins. The line that connects the poles (not shown) is the true quantization axis. Thirdly, the general point on the spin surface corresponds to a mixture of the two eigenstates in the superposition (17). Beyond doubt the Figs. 5 and 6 show that the direction and magnitude of the average spin $\langle\mathbf{S}\rangle$ depend on the contribution of individual eigenstates in the mixed 


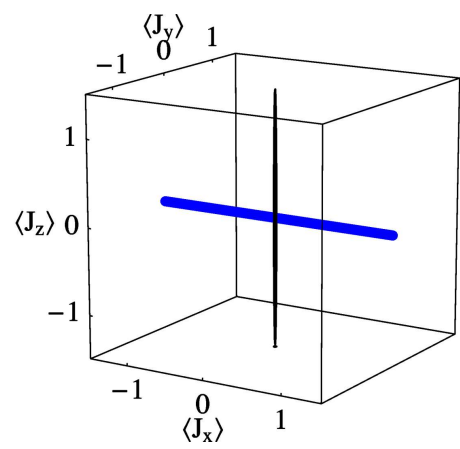

(a)

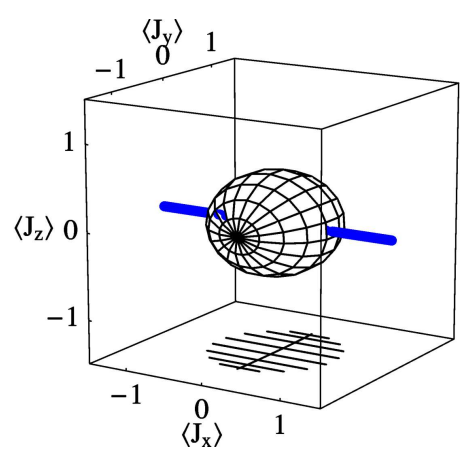

(d)

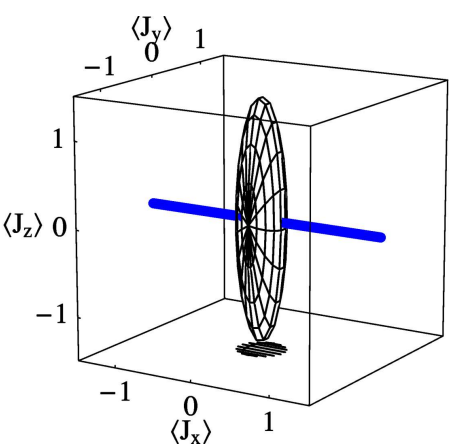

(b)

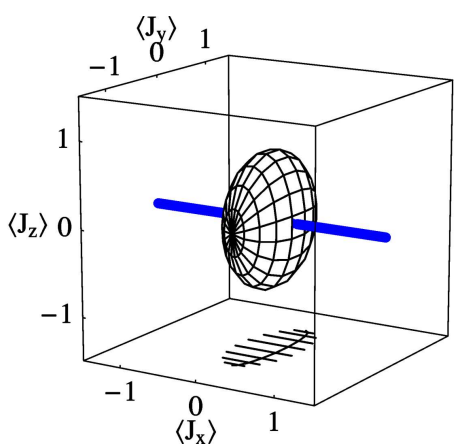

(e)

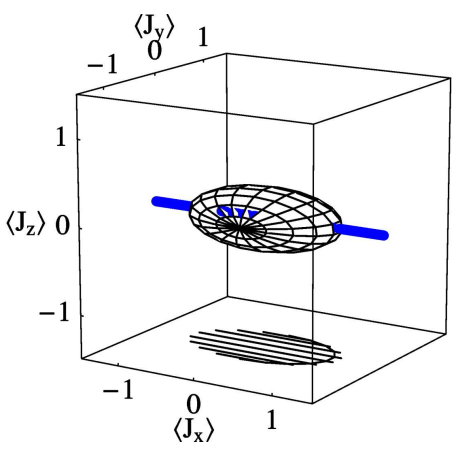

(c)

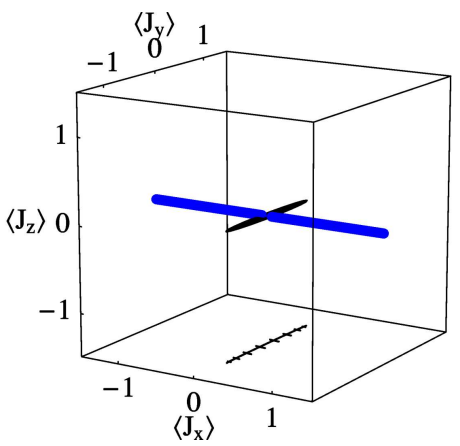

(f)

Fig. 5. The spin surfaces and their projection on $\left\langle J_{x}\right\rangle-\left\langle J_{y}\right\rangle$ plane of (a, b, c) HH1 and (d, e, f) LH1 subbands in $\mathrm{Al}_{0.21} \mathrm{Ga}_{0.79} \mathrm{As} / \mathrm{GaAs}$ QW. The direction of the wave vector is shown by lines: $k_{y}=0$ and (a,d) $k_{x}=0.003$, (b, e) 0.012 , (c, f) $0.024 \AA^{-1}$. The respective wave vectors are designated by letters on the dispersion curves in Fig. 3, too. The QW lies in the $x-y$ plane.

state. The complicated dependence of the spin surface on the wave vector can be explained by different contributions of heavy- and light-mass bands. At $k=0$ these bands are decoupled from each other. However, away from the $k=0$ point the wave function of HH1 band more and more acquires the light-band character and, vice versa, the LH1 band acquires the heavy-band character [27].

In the spin-FET proposed by Datta and Das the contributions of up and down states in the mixture (17) are equal, that corresponds to $\vartheta=\pi / 4$. In Figs. 5 and 6 the corresponding points lie on the equator at equal distances from the poles. When the hole propagates in the FET channel, the tip of $\langle\mathbf{S}\rangle$ moves on the equator. The parameter $\phi$ fixes the initial spin polarization (horizontal or vertical) of the injected hole, similarly as in the superposition (4). It can be shown that in the case of $4 \times 4$ valence Hamiltonian (9) one has $\langle\mathbf{S}\rangle=\langle\mathbf{J}\rangle / 3$, i. e., the shapes of the spin and total angular momentum surfaces apart from their magnitude are identical in this approximation.

The important message in 2D hole case is that the magnitude of the average spin strongly depends on the hole wave vector and even can be equal to zero. This is at variance with the standard belief that in mixing the states with different ratios only spin direction changes. Similar dependences were observed for other directions of $\mathbf{k}$. Comparison between Figs. 5 and 6 shows that the well depth has small effect on the form of spin surface and the strongest dependence comes from the length of the in-plane wave vector $|\mathbf{k}|=\sqrt{k_{x}^{2}+k_{y}^{2}}$. Singularities appear in those cases where the spin surfaces reduce to lines. For example, this happens at $k=0$ in the HH1 subband. In this case the injection from the ferromagnetic contacts with spins polarized in the QW plane will be hard, since the spin lengths in the ferromagnetic source / drain and 2D channel do not match each other. However, for perpendicular polarization the spin matching, in principle, is possible. In the case of LH1 subband an opposite situation is observed, where at $k=0.024 \AA^{-1}$ line-shaped spin surface lies in the QW plane. Thus, in this case an efficient injection will occur if the source and drain magnetization lies in the QW plane. If in the initial Hamiltonian (9) more than four bands are included, the mentioned singular lines will blow up to cigar-shaped spin surfaces. 


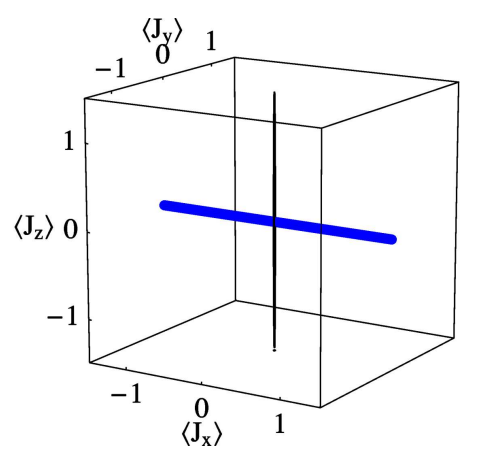

(a)

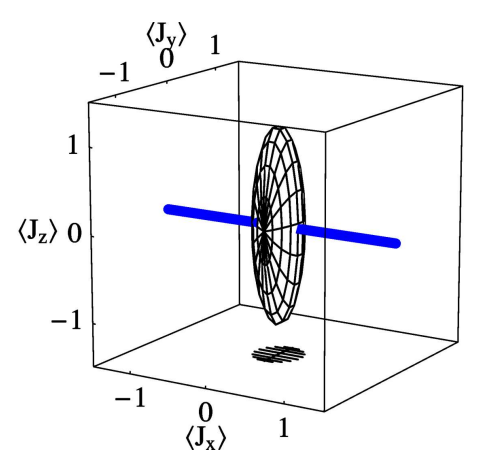

(d)

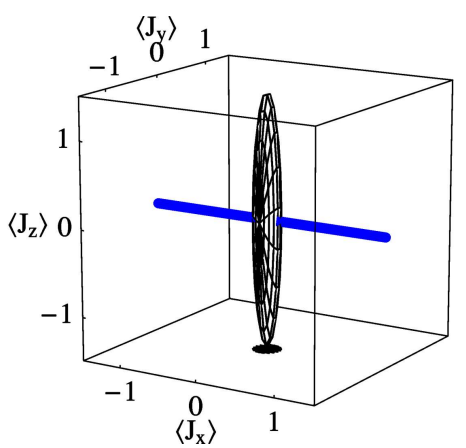

(b)

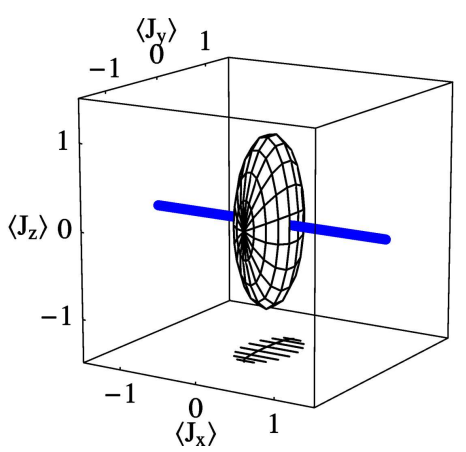

(e)

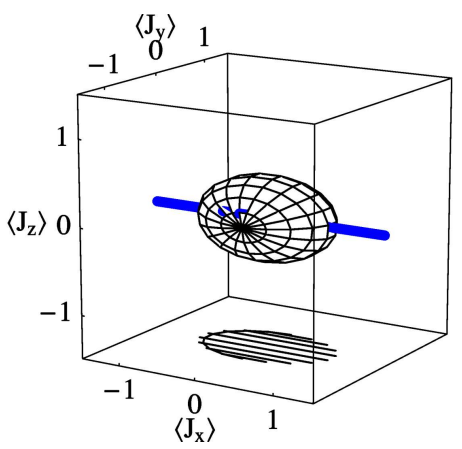

(c)

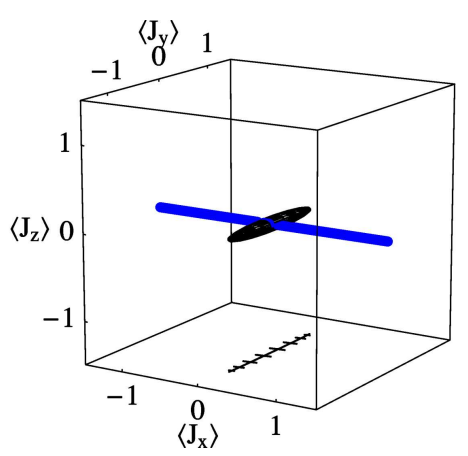

(f)

Fig. 6. The same as in Fig. 5 but for AlAs / GaAs QW.

\section{Conclusion}

In contrast to the standard FET, where only charge transport between the electrodes matters, in the spinFET the properties of the wave function in the channel should be known to describe properly the spin injection, propagation, and collection. The concept of the spin surface allows to solve partially these problems at a quantum mechanical level. Although the spin surface describes spin properties of a freely propagating electron or hole and thus the interface matching problem is circumvented, nonetheless, the knowledge of the spin surfaces in channel and electrodes allows one to make some useful decisions about spin-FET properties. Specifically, the knowledge of the spin surface, first of all, allows one to understand spin properties of the ballistic hole in the channel, where the spin transforms during a carrier flight from source to drain. Second, the interface quality depends on many factors that are frequently unknown to experimenter. The knowledge of the spin surfaces allows one to make some conclusions about the spin injection and collection efficiency in the source/drain electrodes. The standard precession Eq. (3) can be used only to describe electron spin dynamics on spherical spin surface and is not suitable for holes. If needed, the problem may be further simplified by neglecting the Rashba Hamiltonian which does not influence the form of the spin surface. Since it is the Rashba Hamiltonian that takes into account the SO interaction, which is equivalent to some effective magnetic field, in the latter case the information about the direction of the quantization axis will be lost.

In conclusion, a number of important parameters that determine the operation of the spin-FET were listed and analysed critically. The paper shows that this list is not complete and should be supplemented by properties of the spin surfaces which have been found to be rather complicated in the case of $2 \mathrm{D}$ channels. There is a hope that the optimization of spin-FET parameters listed in Sec. 2 will be more fruitful if in the analysis one also takes into account the shapes of spin surface.

\section{References}

[1] S. Datta and B. Das, Electronic analog of the electrooptic modulator, Appl. Phys. Lett. 56(2), 665-667 (1990).

[2] Y.A. Bychkov and E.I. Rashba, Oscillatory effects and the magnetic susceptibility of carriers in inversion layers, J. Phys. C 17(33), 6039-6045 (1984).

[3] R. Winkler, Spin-Orbit Coupling Effects in TwoDimensional Electron and Hole Systems (SpringerVerlag, Berlin-Heidelberg-New York, 2003). 
[4] A. Dargys, Hole spin surfaces in $\mathrm{A}_{3} \mathrm{~B}_{5}$ semiconductors, Phys. Status Solidi B 241(13), 2954-2961 (2004).

[5] A. Dargys, Spin of valence-band holes in wurtzite semiconductors, Phys. Rev. B 72(4), 045220-1-10 (2005).

[6] A. Dargys, Semiconductor spintronics: Role of the valence-band holes, Acta Phys. Pol. A 107(1), 46-55 (2005).

[7] A. Dargys, Spin properties of lead chalcogenides in absence of magnetic field, Phys. Scripta 74(6), 519-524 (2006).

[8] A. Dargys, Precession trajectories of the hole spin in zinc-blende semiconductors, Solid-State Electron. 51(1), 93-100 (2007).

[9] A. Dargys, Spin and orbital motion surfaces in HgTe, Semicond. Sci. Technol. 22(5), 497-501 (2007).

[10] R. Winkler, Rashba spin splitting in two-dimensional electron and hole systems, Phys. Rev. B 62(7), 42454248 (2000).

[11] C.P. Slichter, Principles of Magnetic Resonance (Springer-Verlag, Berlin-Heidelberg-New York, 1980).

[12] A. Dargys, Free-electron spin surfaces in 3D and 2D zinc-blende semiconductors, Phys. Status Solidi B 243(8), R54-R56 (2006).

[13] I. Žutić, J. Fabian, and S.D. Sarma, Spintronics: Fundamentals and applications, Rev. Mod. Phys. 76(2), 323-410 (2004).

[14] F. Meier and B.P. Zakharchenya, Optical Orientation (North-Holland, Amsterdam-New York-Tokyo, 1984).

[15] T. Jungwirth, J. Sinova, J. Mašek, J. Kučera, and A.H. MacDonald, Theory of ferromagnetic (III,Mn)V semiconductors, Rev. Mod. Phys. 78(3), 809-864 (2006).

[16] R.H. Silsbee, Spin-orbit induced coupling of charge current and spin polarization (review), J. Phys. Cond. Matter 16(7), R179-R207 (2004).
[17] M.G. Pala, M. Governale, J. Konig, U. Zulicke, and G. Iannaccone, Two-dimensional hole precession in all-semiconductor spin field effect transistor, Phys. Rev. B 69(4), 045304-1-9 (2004).

[18] D. Sprinzak, M. Heiblum, Y. Levinson, and H. Shtrikman, Ballistic transport of holes and phonon replicas in lightly doped GaAs, Phys. Rev. B 55(16), R10185R10187 (1997).

[19] P.H. Song and K.W. Kim, Spin relaxation of conduction electrons in bulk III-V semiconductors, Phys. Rev. B, 66(3), 035207-1-8 (2002).

[20] D.J. Hilton and C.L. Tang, Optical orientation and femtosecond relaxation of spin-polarized holes in GaAs, Phys. Rev. Lett. 89(14) 146601-1-4 (2002).

[21] S. Bandyopadhaya and M. Cahay, Reexamination of some spintronic field-effect device concept, Appl. Phys. Lett. 85(8), 1433-1435 (2004).

[22] V.M. Ramaglia, D. Bercioux, V. Cataudella, G. DeFilippis, and C.A. Perroni, Spin polarization of electrons with Rashba double-refraction, J. Phys. Cond. Matter 16(50), 9143-9154 (2004).

[23] D. Bercioux and V.M. Ramaglia, The spindouble refraction in two-dimensional electron gas, arXiv: cond-mat/ $0502456,1-4$ (2005).

[24] J. Schliemann, J.C. Egues, and D. Loss, Nonballistic spin-field-effect transistor, Phys. Rev. Lett 90(14), 146801-1-4 (2003).

[25] K.M. Jiang, Z.M. Zheng, B. Wang, and D.Y. Xing, Switching effect in spin field-effect transistors, Appl. Phys. Lett. 89(1), 012105-1-3 (2006).

[26] J.M. Luttinger and W. Kohn, Motion of electrons and holes in perturbed periodic fields, Phys. Rev. 97(4), 869-883 (1955).

[27] E.P. O'Reilly, Valence band engineering in strainedlayer structures, Semicond. Sci. Technol. 4(3), 121137 (1989).

\title{
KODE்L NEVEIKIA SUKINIO TRANZISTORIUS?
}

\author{
A. Dargys \\ Puslaidininkiu fizikos institutas, Vilnius, Lietuva
}

\begin{abstract}
Santrauka
Sukinio tranzistorius yra vienas iš spintronikos prietaisų, pasiūlytas dar 1990 m., kurio veikimas grindžiamas unikaliomis laisvojo elektrono ir skylès sukinio savybėmis puslaidininkiuose. Tokiame tranzistoriuje elektrinio laidumo moduliacija, panašiai kaip optiniame poliarizaciniame moduliatoriuje, valdoma elektriniu lauku, pakeičiant elektrono sukinio poliarizaciją. Ryšys tarp elektrinio lauko ir sukinio sukuriamas per sukinio ir orbitos sąveiką. Deja, iki šiol nepavyko pagaminti veikiančio suki-
\end{abstract}

nio tranzistoriaus, nors moduliacijos galimybès buvo pademonstruotos. Pranešime trumpai apžvelgtas tokio tranzistoriaus veikimo principas, pagrindiniai parametrai, lemiantys jo veikimą, ir sunkumai, su kuriais susiduriama, bandant pagaminti tranzistorių. Ypatingas dèmesys kreipiamas i dvimatès protakos sukinio savybes, tam pasitelkiant autoriaus pasiūlytą sukinio paviršiaus koncepciją. Parodyta, kaip sukinio paviršiaus pavidalas priklauso nuo protakos energinio spektro ir balistinès skylès greičio protakoje. Taip pat parodyta, kaip galima pasinaudoti sukinio paviršiais, optimizuojant sukinio injekciją iš feromagnetiko ị dvimatę protaką. 